\title{
A Flexible Wireless System for Preclinical Evaluation of Retinal Prosthesis
}

\author{
Patrick C. Thien, ${ }^{1,2}$ Rodney E. Millard, ${ }^{1,3}$ \\ Stephanie B. Epp, ${ }^{1}$ and David A. X. Nayagam ${ }^{1,4 *}$ \\ ${ }^{1}$ Bionics Institute, 384-388 Albert St., East Melbourne, Victoria 3002, Australia \\ ${ }^{2}$ Department of Medical Bionics, University of Melbourne, Parkville, Victoria 3010, Australia \\ ${ }^{3}$ Department of Otolaryngology, University of Melbourne, East Melbourne, Victoria 3002, Australia \\ ${ }^{4}$ Department of Pathology, University of Melbourne, St. Vincent's Hospital, Victoria 3065, Australia
}

(Received June 30, 2017; accepted August 21, 2017)

Keywords: electrical stimulation, wireless monitoring, freely moving animals, retinal prosthesis, preclinical

In this paper, we present a novel stimulation controller and monitoring system for evaluating the safety and efficacy of implantable neuroprosthetic devices in a preclinical setting. It features a programmable controller designed to be worn in a custom backpack by freely moving feline subjects. A custom controller powered two, modified, 22-channel clinical stimulators simultaneously. The controller and stimulators together weighed $140 \mathrm{~g}$ and measured $85 \times 70$ $\times 35 \mathrm{~mm}^{3}$. Power was supplied from a $3350 \mathrm{mAh}$ lithium-ion battery. A Bluetooth-enabled laptop-PC base station managed up to six systems and allowed the remote adjustment of the stimulation amplitude and automated collection of stimulator telemetry data. The initial application was for the chronic safety testing of a 44-channel retinal prosthesis. Thirteen feline subjects were implanted with a suprachoroidal electrode array, which was then stimulated or monitored continuously for an average of $54.5 \mathrm{~d}$. Batteries were changed twice weekly and electrode impedances were recorded hourly. This allowed broken electrodes and other issues to be quickly identified and addressed. The ability to remotely control the stimulation amplitude minimised the amount of subject handling that was required, likely reducing subject stress. Existing preclinical evaluation systems are either designed for short-term experiments and have many features but limited battery life, or for long-term static stimulation and are long-lived, but with restricted stimulation parameters and channel counts. Here, we have described a system designed to improve the chronic safety testing of electrode arrays. While it was used here with a suprachoroidal retinal implant, it could be readily adapted for other preclinical models requiring continuous, deterministic stimulation over a prolonged period.

\section{Introduction}

The electrical stimulation of neural tissue in preclinical models is essential for research in the field of retinal prostheses, cochlear implants, and deep brain stimulation (DBS) as well as *Corresponding author: e-mail: dnayagam@bionicsinstitute.org http://dx.doi.org/10.18494/SAM.2018.1651 
for developing any sort of neurostimulator. Assessing the safety and viability of such devices requires the stimulation of tissue for long periods of time, which generally necessitates an awake test subject.

While there are many examples of preclinical systems capable of long-term, wireless, electrical stimulation in the literature (Table 1), there are trade-offs between size, features, and battery life. Also, none of these systems provides an integrated solution that combines the control of stimulation with continuous, long-term monitoring. Such a system would be ideal for chronic safety studies as it would allow testing of the worst-case (i.e., nonstop) use of the device in question.

Including continual electrode monitoring in preclinical studies would also allow more accurate assessments of the effect of total energy delivered to the tissue when examining results retrospectively. This would benefit patients in the long term through neuroprosthetic devices with more clearly defined safety limits.

The smallest devices, often used on mice, are generally limited in the flexibility of their stimulation parameters with some having no configurability after the device is constructed. ${ }^{(1)}$ However, because of this simple hardware, they often have excellent battery life, up to several weeks, on very small batteries. ${ }^{(2)}$

Conversely, larger and more complicated devices ${ }^{(3)}$ offer more in terms of flexibility of stimulation parameters and number of stimulation channels, and are capable of recording highquality evoked neural response waveforms but consequently have a battery life of less than one day and are therefore not suited for chronic safety testing. While it would be possible to perform continuous stimulation using a system with a short battery life, such frequent battery changes are time consuming as well as stressful for the subjects.

In this article, we describe the development of a new system (Fig. 1) for the chronic stimulation of medium-sized laboratory animals requiring many stimulation channels. Our requirements for this system were that it: be small enough to be worn by freely moving feline subjects in a specially made jacket; be rugged enough to last 12 weeks of continuous use; be simple to operate; can log stimulation data and be able to operate on battery for at least two days. In terms of stimulation parameters, the system had to be capable of producing stimulation at rates of 50-200 Hz per electrode for phase widths from 25 to $1000 \mu$ s for as many electrodes as could be driven from a single current source.

A further requirement was to be able to provide stimulation to two electrode channels simultaneously against a common return (termed: paired stimulation), as we were interested in the safety of paired stimulation based on the results of a previous clinical trial. ${ }^{(4)}$

The system was utilised to help test the safety of a 44-channel retinal prosthesis driven by two independent stimulators. For this application, 1-mm-diameter platinum electrodes were stimulated with symmetrical biphasic constant-current pulses against a large-surface-area intraocular platinum return electrode (termed: monopolar stimulation). In addition, because the stimulation was expected to be suprathreshold, there needed to be a mechanism for the stimulation intensity to start subthreshold and increase slowly until the desired level was reached. This is to gradually acclimate the subjects to the unfamiliar stimulation sensation and avoid startling them. 


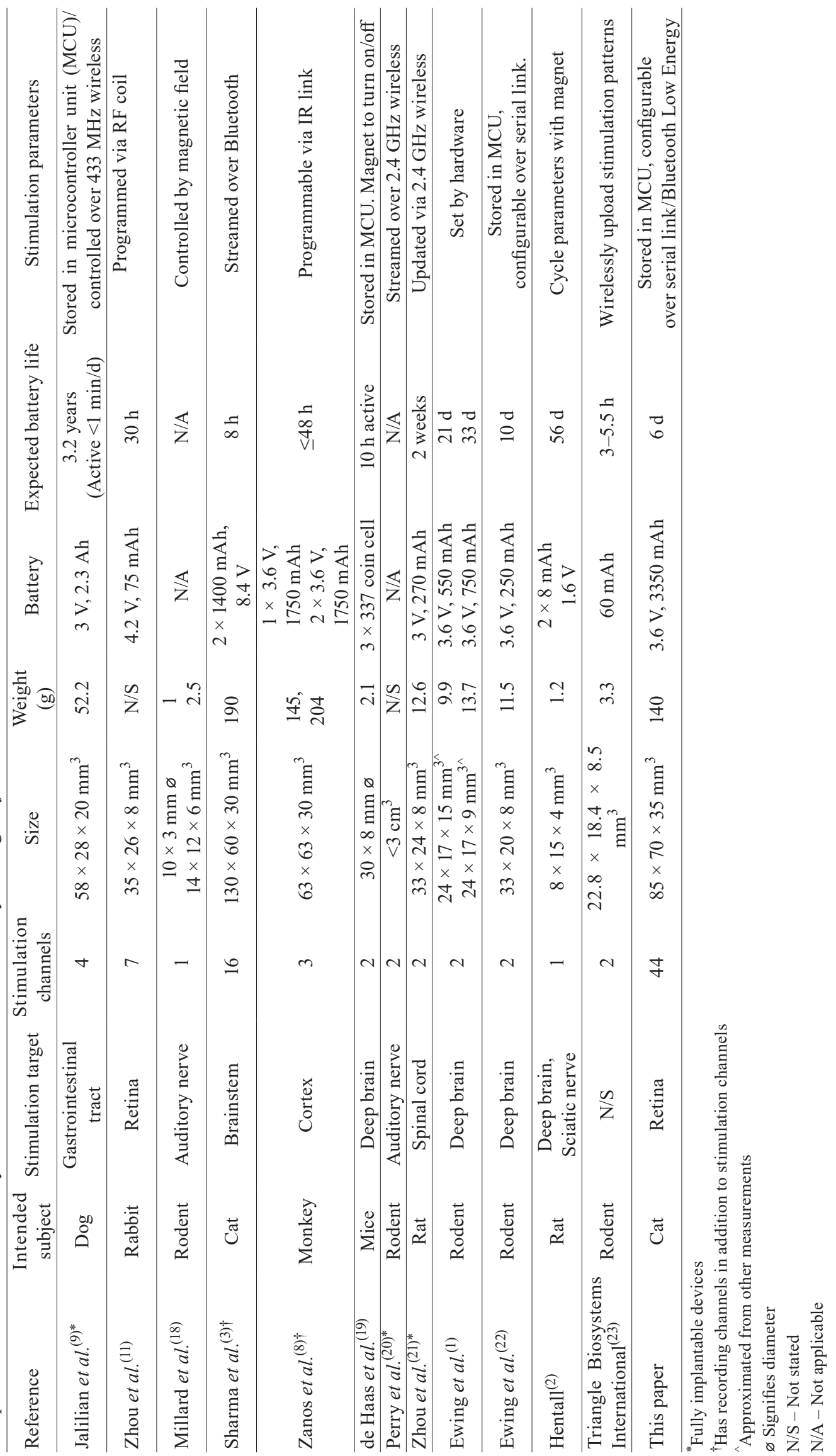




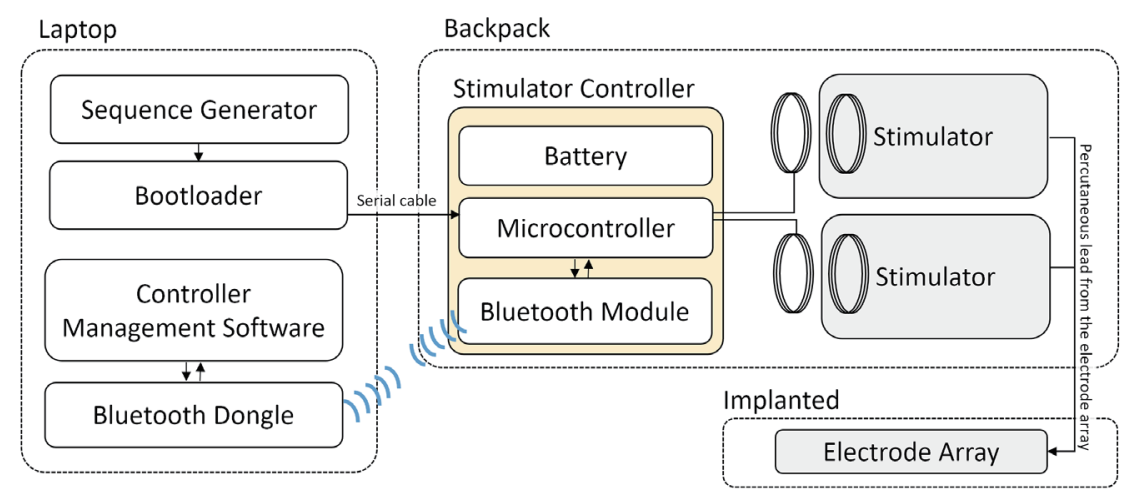

Fig. 1. (Color online) Block diagram of the stimulation system with a single stimulation controller (shaded orange). The system can manage up to 6 controllers. Stimulation sequences are created on the laptop computer using sequence generator software and then a bootloader is used to program the stimulation controller using a serial cable. The stimulator controller sits in a backpack worn by the subject along with two inductively linked stimulators, which are connected via a percutaneous lead to the implanted electrode array. Separate controller management software on the laptop computer is used to manage the controller in the backpack wirelessly via a Bluetooth dongle.

\section{System Description}

\subsection{Stimulator controller hardware}

The stimulator controller includes a 3.3 V PIC microcontroller (PIC24HJ64GP502, Microchip Technology) and a Bluetooth module based on the Texas instruments CC2541 chip (HM-11, JNHuaMao Technology Company) for wireless communications.

The PIC microcontroller's flash memory is used to store the stimulation sequences used for chronic stimulation and transmit them to the clinical stimulators. The CPU clock was configured to run at $20 \mathrm{MHz}$ and derived from the PIC's internal oscillator (specified accuracy: $2 \%$ ). Most instructions take a 1 clock cycle to execute, giving a time resolution of $50 \mathrm{~ns}$ per instruction.

The stimulators used are two commercial multichannel stimulators (Nucleus ${ }^{\circledR}$ CI24RE, Cochlear ${ }^{\mathrm{TM}}$ ), each of which uses a single radio-frequency (RF) coil to transmit power and data. They are controlled by manipulating the digital input-output (IO) pins to produce a $5 \mathrm{MHz}$ on-off keyed waveform and produce biphasic current pulses with a minimum phase duration of $25 \mu$ s and a maximum amplitude of $1.75 \mathrm{~mA}$. They have a compliance voltage of $8 \mathrm{~V}$, and can report voltage measurements with 7.6 bit resolution. The Bluetooth module interfaces with the microcontroller over a serial interface at 2400 baud and notably has very low power consumption in sleep mode.

Power is supplied from a battery containing a rechargeable Panasonic NCR 18650B lithiumion cell coupled with a protection circuit (Blazar NCRB3400mAh). The battery has a nominal voltage of $3.6 \mathrm{~V}$ and a capacity of $3350 \mathrm{mAh}$. The voltage is regulated to $3.3 \mathrm{~V}$ by a buck-boost 
converter (LTC3440, Linear Technology), which has an efficiency of 93-95\% in the operating range for this system.

Components are soldered on a $70 \times 20 \mathrm{~mm}^{2}, 0.8$-mm-thick, 4-layer FR4 printed circuit board (PCB). The height and length were chosen to match the size of the battery, which is attached via conductive clips to the back of the PCB [Fig. 2(b)].

The electronics are protected by a custom case 3D-printed from acrylonitrile butadiene styrene (ABS). A hole in the centre of the PCB allows it to be affixed to the case with a screw. The connectors for the stimulator RF coils are affixed to the body of the case using epoxy and then connected to the PCB using a thin single-core copper wire. The lid of the case, which is held in place by friction (i.e., press-fit), is located on the top to allow for easy access to the battery. On the side of the case is a hole to allow access to a serial port. This is mainly used for reprogramming the device via the bootloader as well as for debugging purposes, and can be plugged with a silicone bung when not in use.

The overall dimensions of the controller in its case are $82 \times 32 \times 25 \mathrm{~mm}^{3}$ and it weighs 70 $\mathrm{g}$, with most of the weight due to the battery $(47.5 \mathrm{~g})$. A stimulator and its RF coil together weigh $34.5 \mathrm{~g}$. When combined, the whole system (controller, $2 \mathrm{RF}$ coils and 2 stimulators) is approximately $85 \times 70 \times 35 \mathrm{~mm}^{3}$ in size and weighs $140 \mathrm{~g}$ [Fig. 2(a)]. It can comfortably sit in the pocket of a custom-made jacket worn by the animals [Figs. 2(c) and 2(d)].

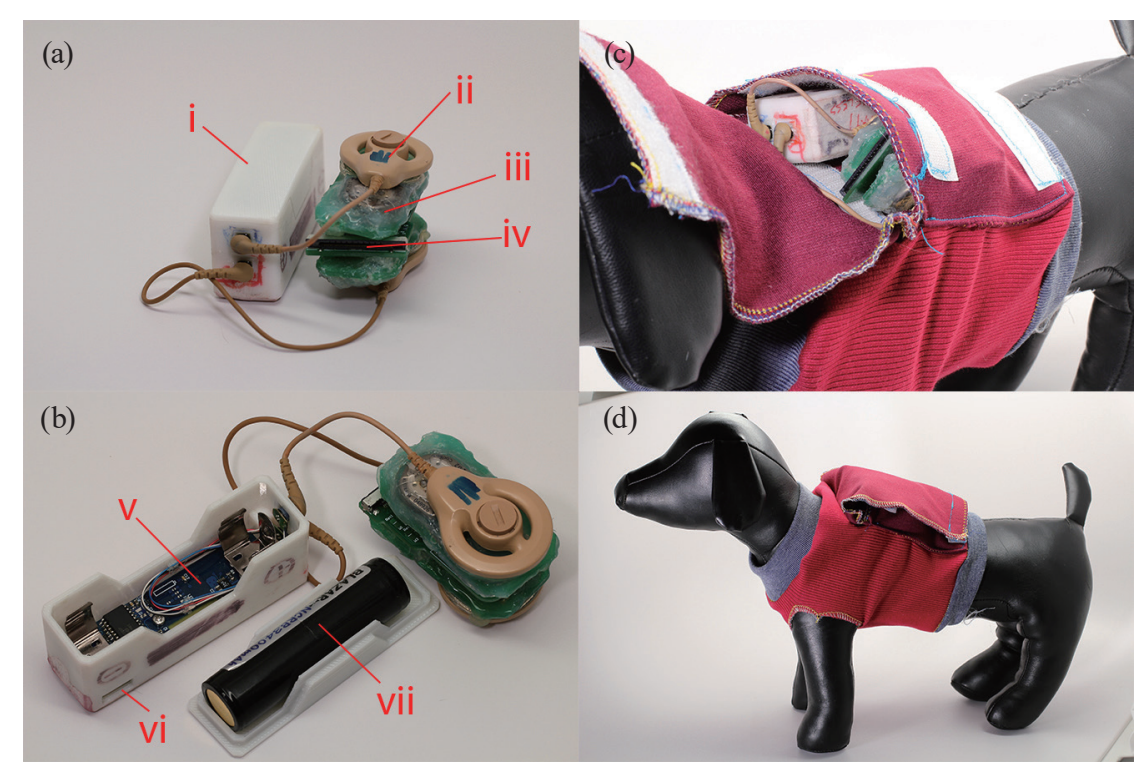

Fig. 2. (Color online) (a) View of a stimulator controller (i) connected to the two stimulators (iii) via magnetically coupled RF coils (ii). The stimulators are connected to the breakout board (iv) that connects to the percutaneous lead from the electrode array. (b) Stimulator controller with the battery (vii) removed and resting on the lid, exposing the main PCB (v). The programming port (vi) can be seen on the side of the controller's case. (c) Looking into the opening of the specially designed backpack to show the stimulator controller and stimulators. (d) Side view of backpack with the components inside and the flap closed. Not shown here is the percutaneous lead that would exit through the skin on the back of the subject's neck and connect the electrode array to the breakout board to which the stimulators attach. 


\subsection{Microcontroller firmware}

Firmware was written in embedded $\mathrm{C}$ and assembly language using the MPLAB ${ }^{\circledR} \mathrm{X}$ IDE (Microchip Technology) and compiled using the XC16 compiler (v1.24, Microchip Technology). The PIC24 Bully Bootloader ${ }^{(5)}$ was installed on each microcontroller as it allowed for quicker reprogramming of the device compared with using the Pickit ${ }^{\mathrm{TM}} 3$. The loading of compiled hex files was carried out using a USB-Serial cable (FTDI TTL-232R-5V-WE).

The microcontroller program was designed to continually produce stimulation by looping over a sequence of pulses stored in memory. On start-up, the controller waits for a signal from the host computer to enable stimulation. Once stimulation is enabled, the program performs an initialisation routine to power up the stimulators, which involves sending a series of null pulses. The program then enters the main loop (Fig. 3), which includes the collection of telemetry from the stimulators. If telemetry was expected but is not received, this indicates that a stimulator has become disconnected and the main loop exits and waits until it is connected again.

The stimulation parameters are stored in two parts; the first contains the pulse data-all the pulse parameters and timing information for each unique pulse in the sequence. The second

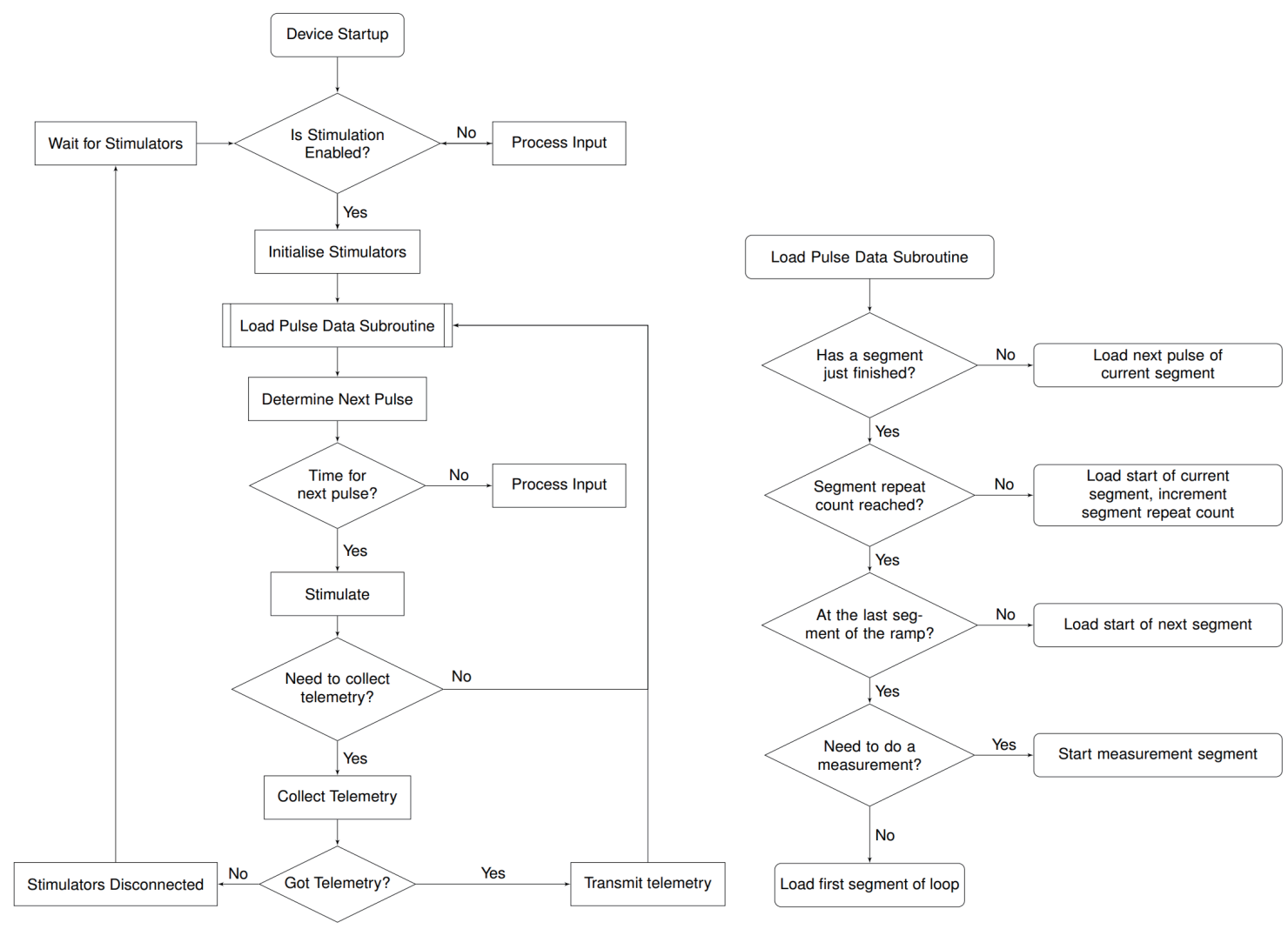

Fig. 3. Flow chart of the program flow in the microcontroller firmware. The main loop of the program checks for a command from the host computer, loads the data for each pulse (the subroutine on the right), waits for the right time before sending it to the stimulators, and then collects and transmits telemetry from the stimulators if necessary. Once the telemetry (if any) is transmitted, the next pulse is loaded. 
part contains the pulse sequence- the order in which the pulses are presented. This serves as a rudimentary compression system; if the same pulse is in the sequence twice, then the data does not have to be duplicated.

The stimulation sequence we used consisted of one set of electrode pairs stimulated in an interleaved fashion for $1 \mathrm{~s}$; then for the next second, a different set of electrode pairs would be stimulated. This would then repeat, such that while one set was stimulating, the other was not. In addition, the stimulation amplitude started low, and after the stimulation was started, the amplitude increased every second until the target was reached. This ensured that the stimulation did not jump quickly above the subjects' perceptual threshold. A simplified version of this is shown in Fig. 4.

To achieve this, the stimulation pulse sequence is broken up into several segments, each with an associated repeat count. Each segment contains several pulses that are presented in order as many times as is specified in the repeat count, before moving on to the next segment. Once the final segment is reached, stimulation continues from the second last segment. Repeating the final segments allows for one or more stimulation parameters (in this case, pulse amplitude) to be gradually increased until a target is reached. Continuing from the second last segment allows for the duty cycle to continue after the ramping period has ended.

In addition to the segments of the main sequence, there are segments used to issue commands to the stimulators in order to collect electrode impedance measurements and other telemetry data from the stimulators. These segments contain the data that is to be sent to the stimulators, for the collection of the required telemetry data, and are used only when requested by the host computer.

The measurement segments are generally short, lasting in the order of tens of milliseconds, and are only inserted into the sequence upon request from the host computer. As such, they do not interrupt the main stimulation sequence for long.

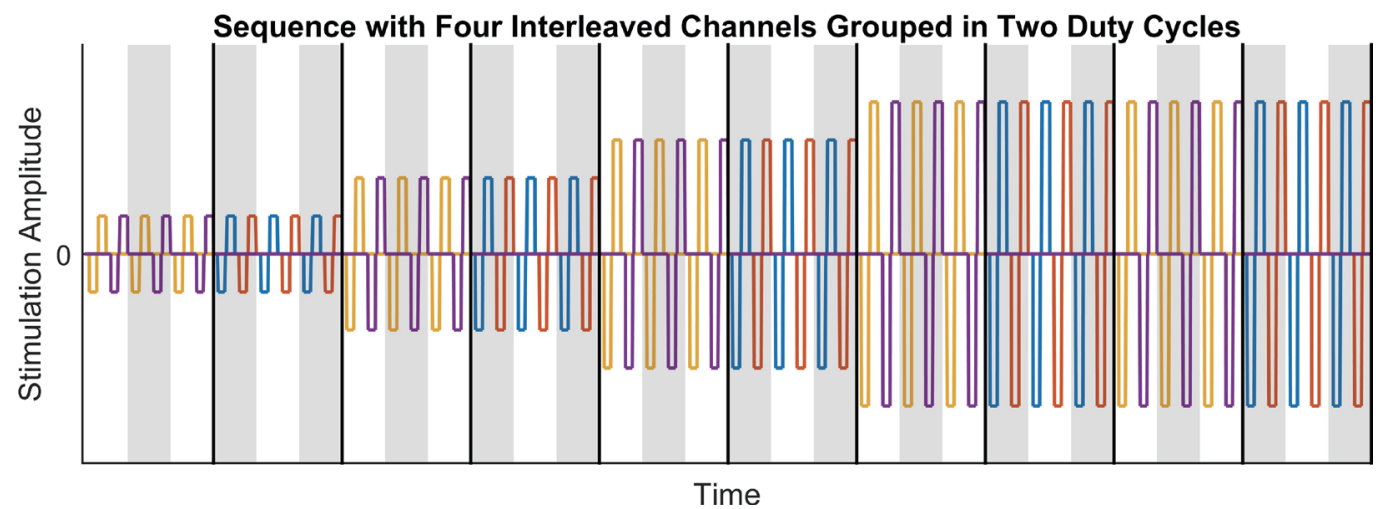

Fig. 4. (Color) Demonstration of stimulation sequences for four channels (yellow, purple, orange, and blue) from one stimulator made up of several segments, each with a repeat count of 3 . The white and grey shaded areas represent repeats of single segments with the bold black lines representing the transition from one segment to the next. Each segment contains two pulses, purple and yellow, or blue and orange. This produces the effect that one set of pulses stimulates for a period of time, then the other set stimulates. Every second segment, the amplitude increases until the target amplitude is reached. At this point, the final two segments are repeated continuously, still maintaining a repeat count of 3 , so the duty cycle continues. 
The segment that the program uses as the final segment of the sequence is wirelessly configurable. This enables the maximum stimulation amplitude to be remotely adjusted. On start-up, this value is initialised to the first segment and stimulation is disabled. Whenever the controller is power-cycled, stimulation must be manually initiated using the management software. This helps to prevent accidental stimulation at levels higher than the subject will tolerate.

\subsection{Host computer software}

\subsubsection{Sequence generation}

An interface was developed to ease the creation of stimulation sequences. This consisted of a Microsoft Excel worksheet in which the sequence segments and segment repeat counts are defined (Fig. S1). The user has full control over the parameters for each pulse including: interpulse interval, phase duration, interphase gap, amplitude, and active and return electrodes for each stimulator; the only restriction being that the timings (interpulse interval, phase duration and interphase gap) are common to both stimulators. At any time, either a single stimulator can be stimulating an active electrode against a return, or both stimulators can be stimulating different active electrodes against a common return to create a pair.

The worksheet contains Visual Basic for Applications (VBA) macros that encode the pulse data and generates the $\mathrm{C}$ source and header files that store the pulse parameters and sequence details in the microcontroller firmware. These source files are compiled into the hex binary that is then loaded onto the controller via a bootloader. Additional macros log details of the sequence being compiled for record-keeping purposes and to assist with the analysis and interpretation of results.

\subsubsection{Controller management}

Also created was the Controller Manager, which is a custom C\# program that communicates with the processors via a Bluetooth dongle (HM-15, JNHuaMao) emulating a serial port (Fig. S2). A single instance of the manager program can control up to 6 processors, which is a limit imposed by the firmware of the Bluetooth dongle used.

The functions of the Controller Manager are to collect regular impedance measurements and to allow the user to wirelessly increase the stimulation amplitude. Collection of impedance measurements can be set up to run automatically at regular intervals or can be triggered manually. With the default settings, impedances were measured once an hour and, at each hour, every measurement was repeated 6 times. The received measurement data is stored in text files on the laptop hard drive for later analysis. The program contains a window for viewing the past measurements for each subject (Fig. S3). The user can select the subject, measurement timepoint, pulse phase duration, and type of measurement (monopolar or common ground) to view.

The Controller Manager can also configure and adjust which segment the controller considers the end of the main sequence, and through this adjust the steady-state stimulation 
amplitude (Fig. S4). This process does not involve changing any of the pulse parameters that were stored when the stimulation controller was programmed. The adjustment of stored pulse parameters requires a wired connection to the programming port.

When stimulation is enabled, stimulation starts with the first segment (which has the smallest amplitude) and continues until the last segment is reached. The variable that indicates which segment the program should consider the last segment is stored in volatile memory, and so is reset when the device is power-cycled.

\subsection{System testing results}

The average power consumption was estimated by connecting the stimulators to an LED test array acting as a dummy load and letting the system run with a fully charged battery until it was fully discharged. The stimulation sequence consisted of $50 \mathrm{~Hz}$ stimulation on 5 pairs of electrodes for a total of $250 \mathrm{~Hz}$ stimulation rate for each stimulator, which is representative of what was used in vivo. Pulses had an amplitude of $625 \mu \mathrm{A}$, a phase width of $400 \mu \mathrm{s}$, and an interphase gap of $20 \mu \mathrm{s}$. The impedance of the dummy load was measured once per hour as it would have been for a real load to ensure that the power consumption of the Bluetooth module was accounted for. With this setup, the battery lasted for $164.3 \mathrm{~h}$. Taking into account the battery's nominal capacity of $3350 \mathrm{mAh}$ and nominal voltage of $3.6 \mathrm{~V}$, this results in an average power consumption of $73.4 \mathrm{~mW}$ for the entire system.

The PIC's internal oscillator controlled all pulse timings. The output pulse rate was tested using an oscilloscope (Agilent MSO-X 3024A) for a programmed stimulation rate of $1000 \mathrm{~Hz}$ on one electrode. For $n=4$ stimulation controllers, the average actual stimulation rate was 992 $\mathrm{Hz}(\max , 993 \mathrm{~Hz}$; min, $989 \mathrm{~Hz}$ ). This indicated that the PIC's oscillator was on average $0.8 \%$ slower than desired, which was within its stated accuracy of $2 \%$. The measurement of pulse width gave a similar result, with $50 \mu$ s pulses being $0.6 \%$ longer than programmed (max, 50.4 $\mu \mathrm{s} ; \mathrm{min}, 50.2 \mu \mathrm{s})$. Pulse amplitudes were sent digitally to the commercial stimulators; therefore, amplitude accuracy was not thoroughly investigated.

\section{Experimental Procedures}

Our group is interested in providing chronic electrical stimulation to the retina for the preclinical safety testing of a suprachoroidal prosthesis. The system was tested with a 44-channel electrode array using methods described previously ${ }^{(6)}$ in 13 normally sighted cats (weight range: 2.4-6.9 kg). Briefly, the electrode array was implanted in the suprachoroidal space of the left eye with a lead wire exiting percutaneously from the back of the neck. Chronic stimulation began once the subject had recovered from surgery, approximately 2 weeks postimplantation.

Several iterations of the system were used during development and testing. Five subjects had a system with no Bluetooth communication, 6 subjects had a system where the wireless communication was used only for recording telemetry data, and 2 subjects had the final system where the wireless communication was also used to control the stimulation amplitude. 
For the subjects where the wireless communication controlled the stimulation $(n=2)$, the subject was manually restrained on a researcher's lap while the controller and stimulators were connected to the electrode array lead wire and then the subject was released. The stimulation was then turned on and the subject was observed for any adverse response. The subject did not need to be restrained for any increases in stimulation amplitude. For any other changes in the stimulation parameters or battery changes, restraining the subject was required to access the controller in the backpack.

For the other subjects $(n=11)$, the stimulation began when the stimulators and controller were connected. For this reason, the subjects were manually restrained while the stimulation amplitude ramped up, such that it could be quickly disconnected in the case of an unexpected adverse reaction.

For both cohorts, the steady-state stimulation amplitude was increased daily (excluding weekends) by approximately $10 \%$ of the target amplitude until either the target was reached, or the subject did not tolerate any further increases. If the subject reacted adversely to the stimulation, it was reduced to the previously tolerated amplitude until the following day.

For the systems that were capable of it $(n=8)$, electrode impedance was measured every hour (Fig. 5). Measurements were made using a $75 \mu \mathrm{A}$ biphasic current pulse and the voltage

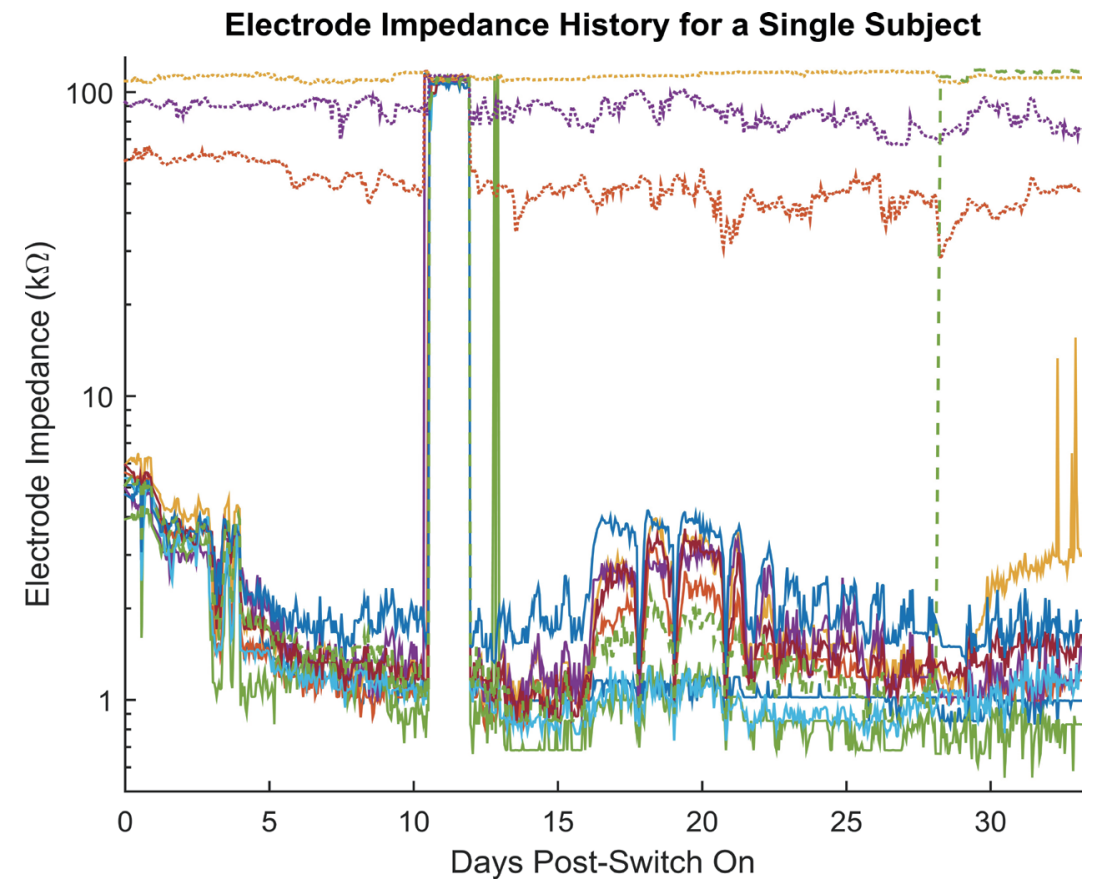

Fig. 5. (Color online) Example of the long-term impedance data captured from the stimulation controller. Six measurements were taken every hour and averaged. Impedance was calculated from the voltage at the end of the first phase of a biphasic current pulse with a phase width of $25 \mu \mathrm{s}$, divided by the nominal current of $75 \mu \mathrm{A}$. Owing to compliance voltage limitations of the stimulators, open-circuit electrodes show an impedance of approximately $120 \mathrm{k} \Omega$. Three electrodes were unusable from the start due to being open-circuit (yellow-dotted line) or high impedance (orange and purple dotted lines). One electrode (dashed line) failed 28 days after stimulation began. The remaining electrodes (solid lines) worked for the entire experiment. The jump in electrode impedances on days $10-12$ is due to the percutaneous lead wire becoming disconnected. 
was taken at the end of the first phase. This was done for both a monopolar and common ground return configuration. Impedances in the monopolar return configuration were also measured using pulses of various phase durations $(25,145,290,400$, and $580 \mu \mathrm{s})$. This allowed the voltage waveform to be reconstructed for later analysis (Fig. 6).

For the other, earlier, systems $(n=5)$, impedance measurements were made approximately every two weeks and involved manually restraining the subject on a researcher's lap throughout the duration of the measurement process. This process is similar to the one used by Nayagam et $a l .{ }^{(6)}$ In all cohorts, batteries were changed approximately twice a week to ensure that they were never depleted.

In addition, the number of times the stimulation was adjusted or the subjects had to be handled to access the controller during their experimental period was recorded as summarised in Fig. 7. This shows the impact that the introduction of wireless communications had on the amount of handling required with the subjects. As a control, the number of times each subject was handled to change the battery did not change considerably, although the range of interactions decreased slightly with each iteration of the system as we gained experience with it.

Perhaps surprisingly, the initial addition of wireless communications for electrode monitoring increased the number of times that the stimulation parameters were changed for reasons other than increasing the amplitude. This can be attributed to system faults such as broken electrodes being more readily detectable, and so the stimulation was adjusted more frequently to account for these.

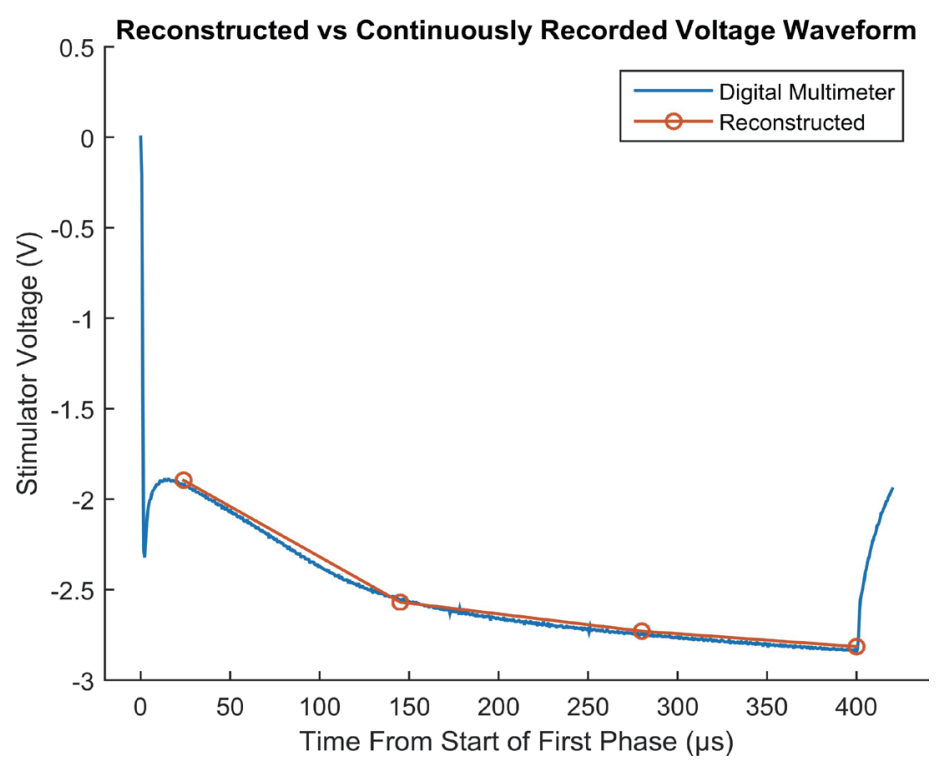

Fig. 6. (Color online) Voltage waveform of the first phase of a $75 \mu \mathrm{A}$ biphasic pulse generated by an optically isolated constant current stimulator, developed in-house, and sampled at $1.8 \mathrm{MHz}$ with a digital multimeter (NI PXI 4072) (blue) (as previously described by John et al. ${ }^{(7)}$ ). This is compared with a waveform reconstructed from data collected by the stimulator controller on the same electrode (orange). Several pulses were sampled at either 25, 145, 290 , or $400 \mu$ s after the start of the first phase, and the voltage waveform was reconstructed by linear interpolation. 


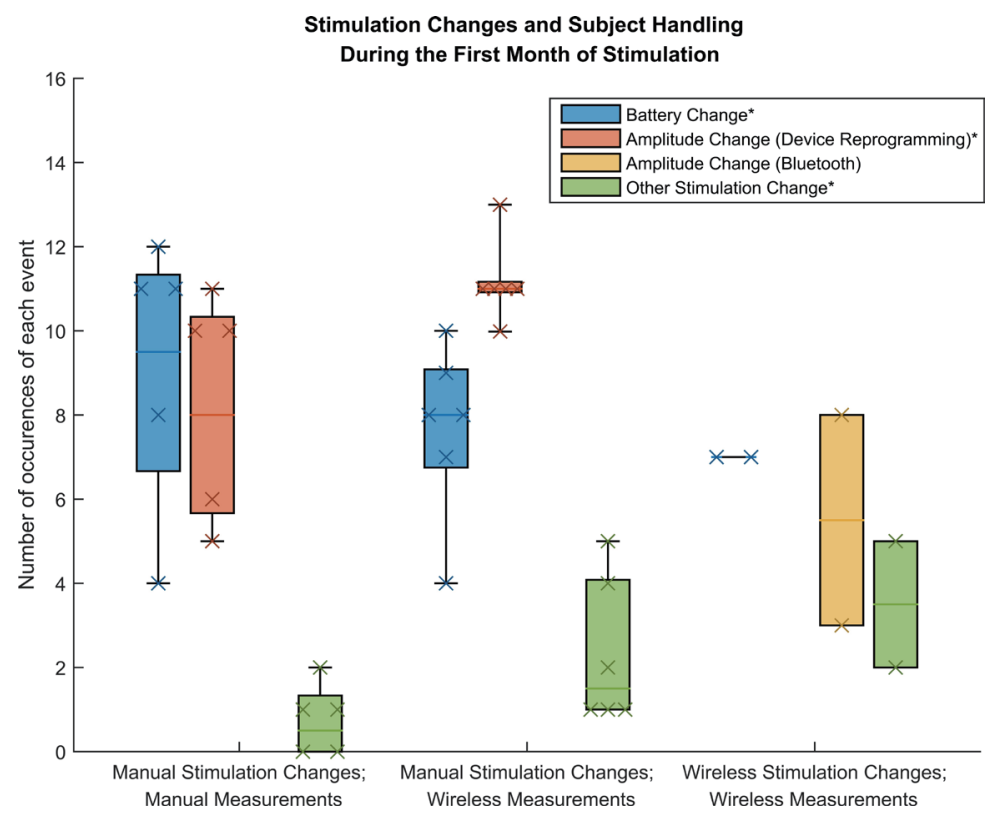

Fig. 7. (Color) Box-and-whisker plots representing the number of times each subject's stimulation was adjusted or battery changed for the three different iterations of the system throughout its development. Data is taken from the first month of stimulation for each subject. Actions in the legend marked with an asterisk required the subject to be held while it was performed. In the first two iterations of the device, changing the amplitude of stimulation required plugging the controller into a computer to reprogram it (orange). In the final iteration, the stimulation amplitude could be changed wirelessly (yellow) whereas changes to other parameters, such as changing electrodes, required reprogramming the controller (green). Whiskers represent the maximum and minimum values and boxes show the first and third quartiles, with the line inside representing the median. Crosses show individual data points.

When the system was updated to allow the stimulation amplitude to be changed wirelessly, the number of adjustments for reasons other than changing the amplitude stayed within the same range, as would be expected. However, as changing the amplitude no longer required reprogramming the controller, the overall number of adjustments requiring the physical handling of the subject was reduced.

In addition, the number of times the amplitude was changed wirelessly is much less than the median of 11 times it was changed when it required reprogramming the controller. This can be attributed to the fact that we were able to increase stimulation more than the originally intended $10 \%$ per day, as amplitude adjustments were much faster without needing to disconnect and reprogram the device. Subjects were also able to freely roam while the amplitude was adjusted

\section{Discussion}

The stimulation system presented here offers a compromise between size, battery life, and flexibility, which is geared towards long-term experiments where the stimulation parameters are not anticipated to change frequently. 
Of the systems listed in Table 1, the systems designed for rats, rodents, and mice are all very light and generally have excellent battery life, but none of them have more than two stimulating channels, which make them unsuitable for the testing of high-channel-count electrode arrays. The system presented here is one of the heavier ones, although it is the lightest one that provides any recording capabilities. Both heavier devices ${ }^{(3,8)}$ have more emphasis on the recording of neural signals than this system, which is not necessary for chronic safety testing. The fully implantable device by Jalilian et al. ${ }^{(9)}$ offers an impressive set of features including wireless control, although were it to be continuously active, the battery would last less than a day.

Because all pulse parameters must be included in the controller's firmware, this system is not capable of real-time stimulation with arbitrary pulses, as is the case in other systems. ${ }^{(3)}$ This was an intentional architectural decision as it removes the complications that come with streaming data such as communications latency, the need to maintain a buffer, data integrity checks, and the reliance on the host PC being continually operational. While these could be mitigated, they come with computational and power overheads that are not necessary for a system that is intended to produce repetitive stimulation. It is simpler to have the wireless communications only for controlling the presentation of parameters already in memory and sending back the relatively small amounts of telemetry data.

The use of clinically approved stimulators reduced the design time and testing overhead of the project considerably, as no custom stimulator hardware needed to be verified. The clinical stimulators have already been rigorously tested. This is beneficial for a safety study, as any damage caused is more likely to be due to the surgery or stimulation itself, rather than a defect in the stimulator.

In the current system, changing the pulse parameters requires physically plugging the controller into a computer so that the firmware can be reprogrammed. A potential future upgrade would be to enable the bootloader to work via Bluetooth, which would enable all stimulation parameters, including the choice of electrodes, to be configured without the need to disconnect and remove the system from the subject. This would further reduce the amount the subjects need to be handled, although it would require some hardware modifications in addition to software changes.

The average stimulation rate used in the in vivo experiments was less than the theoretical maximum the stimulators can produce and therefore the battery lasted longer than the minimum designed lifetime. The extra battery life allowed the battery to be changed less frequently, which reduced the amount of handling the animals required, although future iterations of the system may opt for a smaller battery to reduce the overall size of the system. In addition to reducing the battery size, the system can be configured to use only one stimulator if the additional stimulation channels are not needed, to further reduce the weight and volume.

Having a reprogrammable microcontroller and Bluetooth communications attached to the stimulators provides a flexible hardware base. From this, only software changes are required to reconfigure the system for a variety of different experiments. For example, behavioural experiments could be designed where certain actions by the subject wirelessly trigger the controller to start or stop stimulation. 
The frequent, wireless electrode impedance measurements collected allow for the quick detection of dead batteries, broken electrodes or out-of-compliance stimulation. Consequently, the total stimulation delivered to individual electrodes can be calculated more accurately, as it is recorded automatically at hourly intervals. This is compared to relying on manual checks of the state of the electrodes, which for the first 5 subjects in this study was relatively infrequent, happening at most 8 times in the first month of stimulation (data not shown).

Stimulation with a current-controlled stimulator outside of its compliance voltage can lead to unbalanced stimulation resulting in a net transfer of charge, which can be damaging to tissues. ${ }^{(10)}$ This can happen due to changes in the electrode-tissue interface leading to increases in electrode impedance, or because of the electrode breaking away from the wire it connects to. When detected, stimulation to the affected electrodes should be adjusted. If it is not detected quickly and histological examination of the tissue shows that damage has occurred, it becomes harder to determine if the level of stimulation used is safe, as the damage may have been caused by unwanted DC stimulation.

While chronic safety studies without continuous monitoring ${ }^{(6,11)}$ can, and have, been carried out for preclinical devices, which have then gone on to human clinical trials, ${ }^{(4,12)}$ continuous stimulation coupled with automated monitoring provides the benefit of a much quicker detection of problems. Manually collecting even daily measurements for an implanted device with our system would not only be very time consuming, but likely a significant source of stress for the subjects. The system described by Tashiro et al. ${ }^{(13)}$ allows for the automated collection of impedance data concurrently with stimulation. However, unlike the system described in this paper, theirs is a tethered system, so while it can make much more detailed measurements, the subjects must remain caged while being stimulated.

Having high-temporal-resolution electrode impedance data allows for the total energy dosage delivered to the tissue to be much more accurately calculated. For systems without impedance telemetry capabilities, it is only possible to know the charge injected (for a constantcurrent stimulator), and energy delivery calculations must rely on manually conducted electrode impedance measurements.

In addition, because the voltage waveform can be reconstructed, it is possible to analyse the change in the resistive and capacitive components of the impedance of the electrode tissue interface. This has been studied before both in vivo and in vitro, ${ }^{(14,15)}$ but not at such a high temporal resolution for extended periods of time in a preclinical model. This could potentially lead to improved outcomes for patients as knowledge of temporal changes in the electrodetissue interface and associated safety limitations improves. In particular, finer control over the ohmic heating of tissue will lead to safer stimulation strategies.

The handling of subjects and changes to the stimulation are similar to stressors used elsewhere for cats. ${ }^{(16)}$ While we did not explicitly record any measures of cat stress, ${ }^{(17)}$ we hope that as well as being good for their welfare, the reduced handling of subjects and increased separation of handling and stimulation will reduce any stress responses and make them easier to work with in the future. 


\section{Conclusions}

In summary, we have presented a system for improved preclinical retinal prosthesis safety evaluation. It consists of a flexible stimulator controller capable of controlling two clinicalgrade stimulators on freely moving laboratory animals. The strength of the system lies in its ability to provide long-term monitoring over a low-power wireless communications link. The functional enhancements described here for the purposes of preclinical chronic safety testing will ultimately lead to improved clinical outcomes. Furthermore, this system could easily be adapted for use in other areas of neuroscience, where such long-term stimulation studies are needed.

\section{Financial Declaration}

This research was supported by the Australian Research Council (ARC) through its Special Research Initiative (SRI) in Bionic Vision Science and Technology grant to Bionic Vision Australia (BVA). Further support was provided by a National Health and Medical Research Council Development Grant (APP1120664). The Bionics Institute receives Operational Infrastructure Support from the Victorian Government. The Bionics Institute would also like to acknowledge support from the Bertalli Family Trust and the J T Reid Charitable Trust. The funders played no role in the study design, data collection and analysis, decision to publish, or preparation of the manuscript.

\section{Conflicts of Interest}

The authors declare no conflicts of interest.

\section{Ethics Statement}

All procedures were approved by The Royal Victorian Eye and Ear Hospital's Animal Research \& Ethics Committee (RVEEH AREC:11/244AB, 15/324AB). The subjects were treated according to the National Health and Medical Research Council's "Australian Code of Practice for the Care and Use of Animals for Scientific Purposes" (2013) and the "Prevention of Cruelty to Animals Act" (1986; and amendments). All surgical, clinical assessment, and electrophysiological procedures were carried out under anaesthesia and all efforts were made to minimise suffering.

\section{Acknowledgments}

The authors would like to thank the following people for their kind assistance: Mohit Shivdasani, Peter Seligman, Alice Brandli, Carla Abbott, Owen Burns, Joel Villalobos, Phillip Senn, Dimitra Stathopoulos, and Chris Williams. 


\section{References}

1 S. G. Ewing, W. J. Lipski, A. A. Grace, and C. Winter: J. Neurosci. Methods 219 (2013) 324.

2 I. D. Hentall: Front. Neuroeng. 6 (2013) 8.

3 V. Sharma, D. B. McCreery, M. Han, and V. Pikov: IEEE Trans. Neural Syst. Rehabil. Eng. 18 (2010) 67.

4 L. N. Ayton, P. J. Blamey, R. H. Guymer, C. D. Luu, D. A. X. Nayagam, N. C. Sinclair, M. N. Shivdasani, J. Yeoh, M. F. McCombe, R. J. Briggs, N. L. Opie, J. Villalobos, P. N. Dimitrov, M. Varsamidis, M. A. Petoe, C. D. McCarthy, J. G. Walker, N. Barnes, A. N. Burkitt, C. E. Williams, R. K. Shepherd, P. J. Allen, and Bionic Vision Australia Research Consortium: PLoS One 9 (2014) e115239.

5 R. Reese, B. A. Jones, and J. W. Bruce: Pic24 Bully Bootloader, http://www.reesemicro.com/Home/pic24software-library-collection/pic24-bully-bootloader (accessed January 2017).

6 D. A. X. Nayagam, R. A. Williams, P. J. Allen, M. N. Shivdasani, C. D. Luu, C. M. Salinas-LaRosa, S. Finch, L. N. Ayton, A. L. Saunders, M. McPhedran, C. McGowan, J. Villalobos, J. B. Fallon, A. K. Wise, J. Yeoh, J. Xu, H. Feng, R. Millard, M. McWade, P. C. Thien, C. E. Williams, and R. K. Shepherd: PLoS One 9 (2014) e97182.

7 S. E. John, M. N. Shivdasani, J. Leuenberger, J. B. Fallon, R. K. Shepherd, R. E. Millard, G. D. Rathbone, and C. E. Williams: J. Neural Eng. 8 (2011) 036011.

8 S. Zanos, A. G. Richardson, L. Shupe, F. P. Miles, and E. E. Fetz: IEEE Trans. Neural Syst. Rehabil. Eng. 19 (2011) 427.

9 E. Jalilian, D. Onen, E. Neshev, and M. P. Mintchev: Med. Eng. Phys. 29 (2007) 238.

10 R. K. Shepherd, N. Linahan, J. Xu, G. M. Clark, and S. Araki: Acta Oto-Laryngologica 119 (1999) 674.

11 J. A. Zhou, S. J. Woo, S. I. Park, E. T. Kim, J. M. Seo, H. Chung, and S. J. Kim: J. Biomed. Biotechnol. 2008 (2008) 547428.

12 T. Fujikado, M. Kamei, H. Sakaguchi, H. Kanda, T. Morimoto, Y. Ikuno, K. Nishida, H. Kishima, T. Maruo, K. Konoma, M. Ozawa, and K. Nishida: Invest. Ophthalmol. Visual Sci. 52 (2011) 4726.

13 H. Tashiro, Y. Terasawa, M. Kuwabara, K. Osawa, T. Tokuda, J. Ohta, and T. Fujikado: Adv. Biomed. Eng. 6 (2017) 8.

14 C. Newbold, S. Mergen, R. Richardson, P. Seligman, R. Millard, R. Cowan, and R. Shepherd: Cochlear Implants Int. 15 (2013) 191.

15 C. Newbold, R. Richardson, R. Millard, C. Huang, D. Milojevic, R. Shepherd, and R. Cowan: J. Neural Eng. 7 (2010) 056011.

16 K. Carlstead, J. L. Brown, and W. Strawn: Appl. Anim. Behav. Sci. 38 (1993) 143.

17 M. E. Gruen, A. Thomson, G. Clary, A. Hamilton, L. Hudson, R. Meeker, and B. Sherman: Lab Anim. 42 (2013) 385.

18 R. E. Millard and R. K. Shepherd: J. Neurosci. Methods 166 (2007) 168.

19 R. de Haas, R. Struikmans, G. van der Plasse, L. van Kerkhof, J. H. Brakkee, M. J. Kas, and H. G. Westenberg: J. Neurosci. Methods 209 (2012) 113.

20 D. W. J. Perry, D. B. Grayden, R. K. Shepherd, and J. B. Fallon: J. Neural Eng. 9 (2012) 014001.

21 H. Zhou, Q. Xu, J. He, H. Ren, H. Zhou, and K. Zheng: J. Neurosci. Methods 204 (2012) 341.

22 S. G. Ewing, B. Porr, J. Riddell, C. Winter, and A. A. Grace: J. Neurosci. Methods 213 (2013) 228.

23 Triangle Biosystems International: Neural Stimulator Systems, http://www.trianglebiosystems.com/s-seriessystems.html (accessed July 2017). 
Appendix

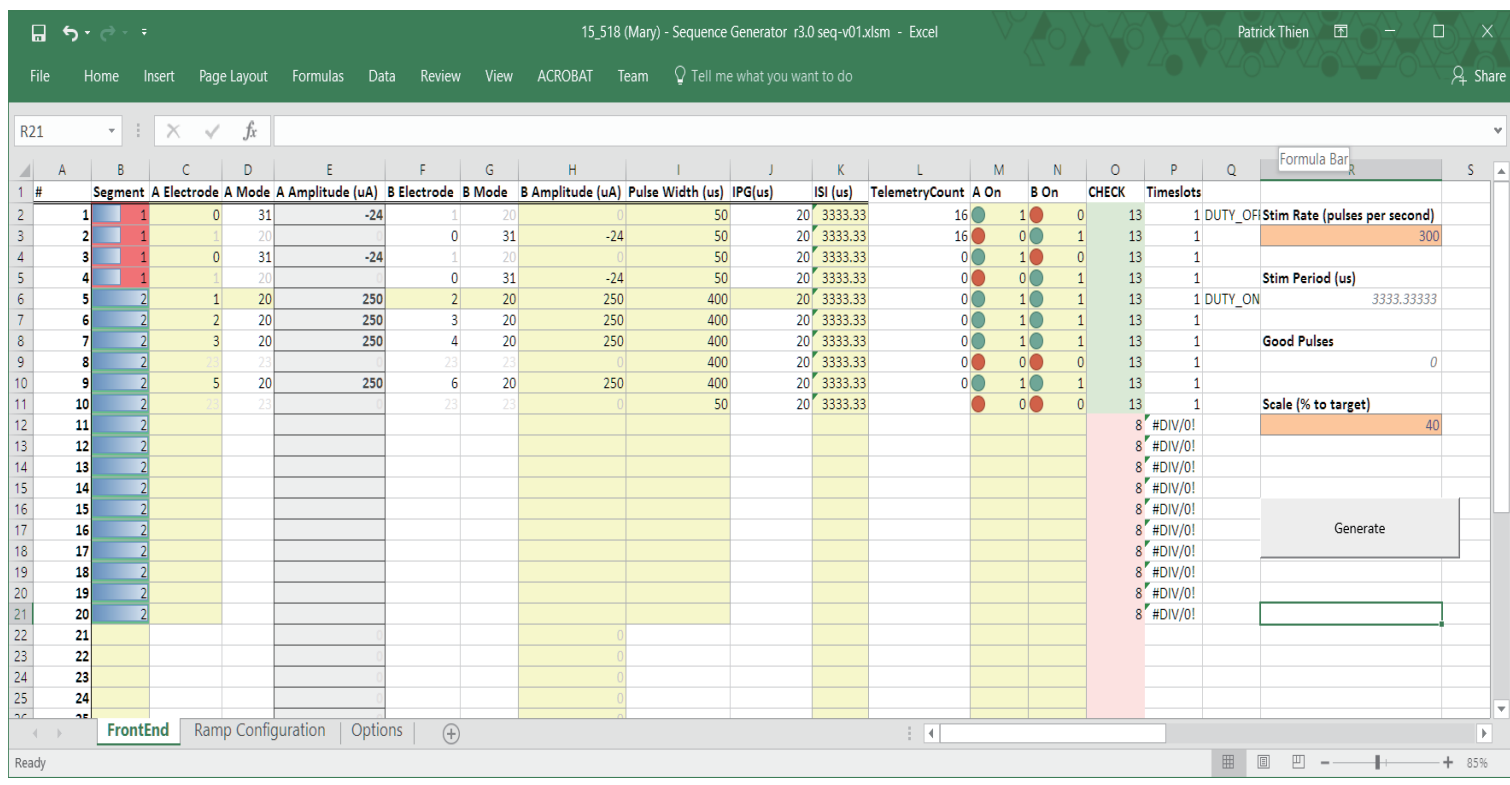

Fig. S1. (Color online) A screenshot of the Excel sheet used to enter the stimulation sequence. The data for each pulse in the sequence is entered in columns B-N. Only two segments need to be entered, and these are considered to be the final segments of the sequence containing the target amplitude. Macros automatically expand this into 200 segments, with the amplitude rising by $1 \%$ of the target every two segments. The generate button in the bottom right is used to generate the required source files and calls the compiler.

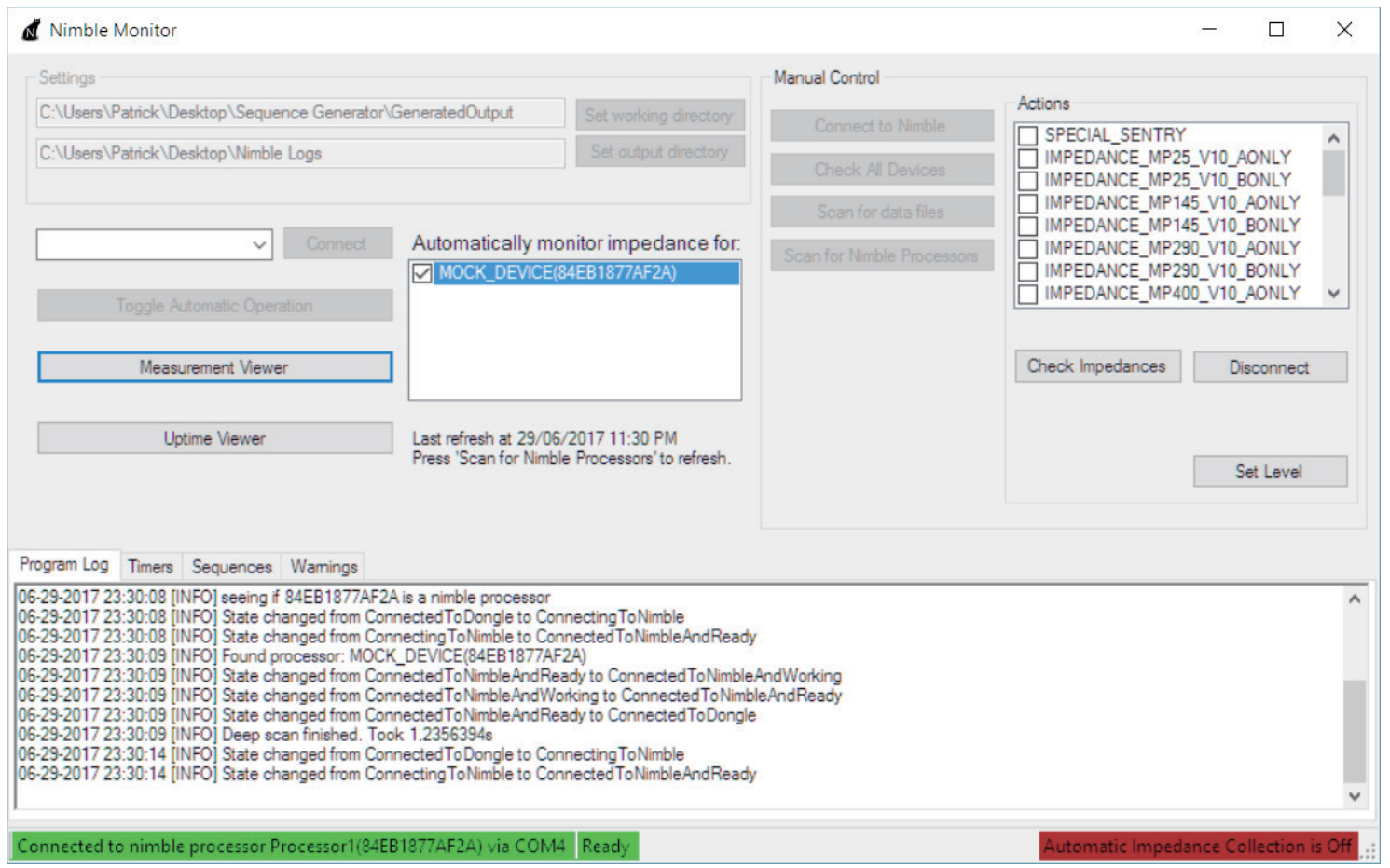

Fig. S2. (Color online) Main window of the controller management software (internal name "Nimble Monitor"). The upper left quadrant of the window contains controls for selecting the working and output directories, connecting to the Bluetooth dongle, and selecting the controller to manage. When "Automatic Operation" has been toggled off, the manual control portion of the window (upper right quadrant) becomes enabled. Here, the user can connect to a single controller, manually initiate an impedance check, or change the stimulation level. The bottom half of the screen contains a scrolling log window for diagnostic purposes. 


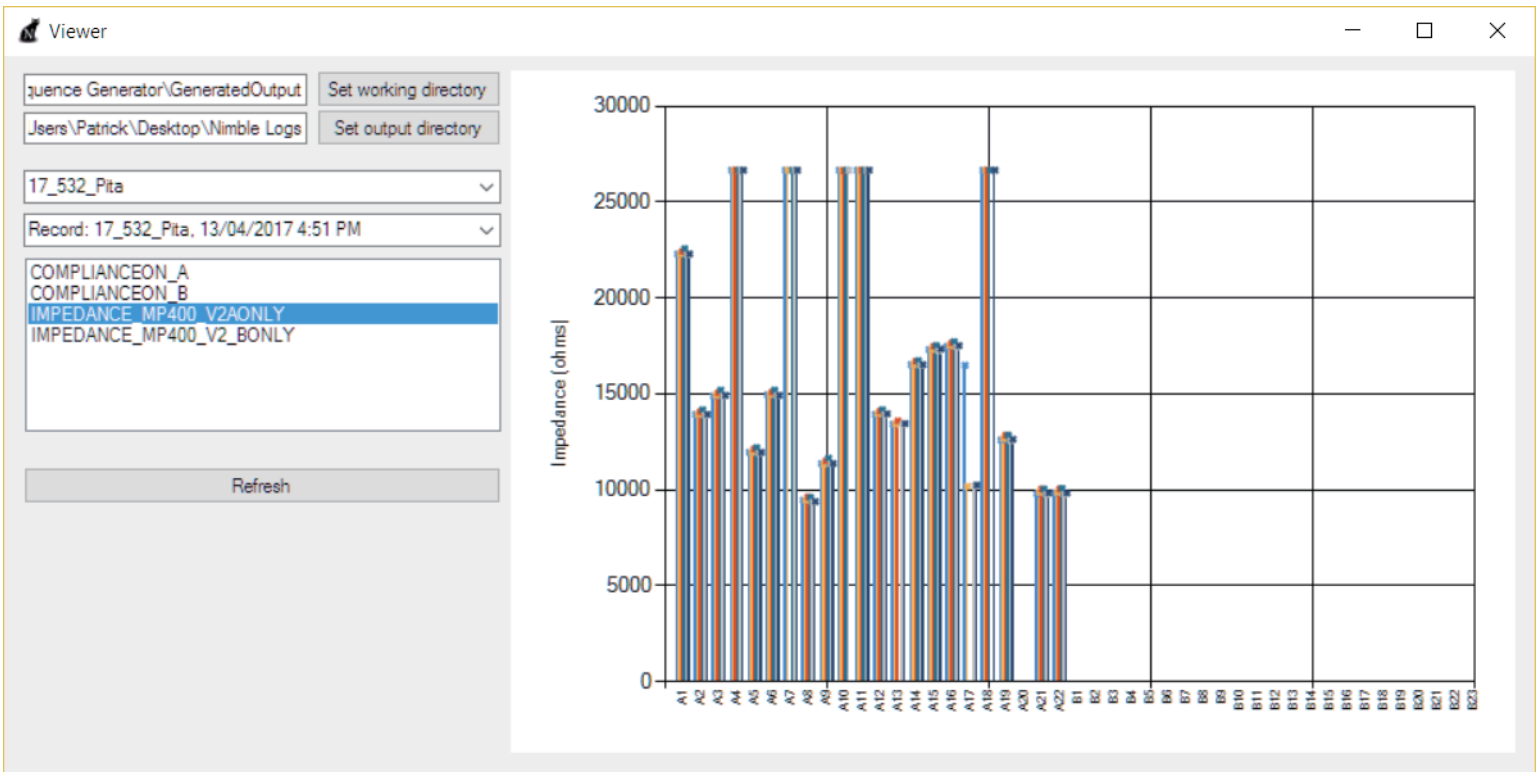

Fig. S3. (Color online) Measurement viewer of the controller management software that can be accessed by clicking the "Measurement Viewer" button on the main view. In this window, users can view the impedance measurements for any subject at any point in time. On the left side are two dropdown boxes, one to select the subject, the other to select the point in time. Below these is a listbox containing the different measurements taken at that timepoint. On the right, a graph of the impedances for the selected measurement is shown.

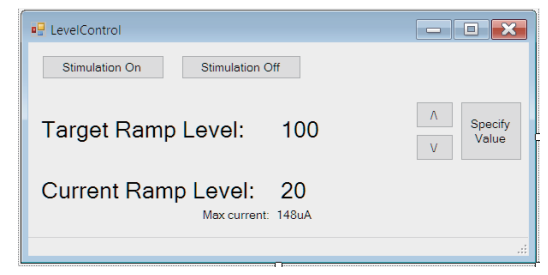

Fig. S4. (Color online) Level control screen of the controller management software that can be accessed by clicking the "Set Level" button on the main view. In this window, users can set the amplitude of the currently connected controller, as well as turning the stimulation on and off. The ramp level refers to the percent of the maximum amplitude of the sequence. The "Max current" field shows the maximum current of the current segment. 